\title{
Revealing Microstructural Defects in Commercial Li-Ion Batteries under Electrochemical Fatigue Cycling
}

\author{
Jeff Gelb $^{1,2}$, Donal Finegan ${ }^{3}$, Dan Brett ${ }^{3}$, and Paul Shearing ${ }^{3}$ \\ 1. San Jose State University, Department of Chemical and Materials Engineering, San Jose, CA, USA \\ 2. Carl Zeiss X-Ray Microscopy, Inc., Pleasanton, CA, USA \\ 3. The Electrochemical Innovation Lab, Department of Chemical Engineering, University College \\ London, London, UK
}

Li-ion batteries serve a range of applications within daily life. From portable electronics to the automotive industry, the global reliance on Li-ion battery technologies is projected to grow at an exponential rate over the next decade. With increasing market demands and widespread application, however, comes the unique responsibility of scientists and engineers to characterize and understand the aging and failure properties of batteries. The aim of this research is to simultaneously satisfy the following consumer demands: a) the materials should be long lasting, particularly important in the automotive industry, and b) devices made with battery materials should be safe to operate. To better address these technological challenges, researchers worldwide are beginning to turn to microscopy to understand how the materials perform in their service conditions and to identify the root cause for failures to mitigate the risk of consumer dissatisfaction and, ultimately, to preserve consumer safety.

The battery systems, however, represent a unique challenge to microscopists and characterization scientists. These systems are typically multi-scale structures, with aging and evolution effects happening from the pack scale all the way down to sub-particle defect nucleation. In order to address the challenges outlined above, a multi-scale investigation approach is needed, to characterize large scale device issues as well as pore- and particle-scale phenomena.

$\mathrm{X}$-ray microscopy $(\mathrm{XRM})$ is a unique technique, capable of producing 3D volumetric data across a range of length scales, from the tens of millimeters down to the tens of nanometers. This technique further offers the advantage of being non-destructive, facilitating 4D studies of aging processes inside packaged batteries over a range of charge conditions, and preserving the specimen for further analysis using correlative microscopy techniques (e.g., with light-, electron-, and ion-microscopy, as well as electronand x-ray microprobe analysis).

Here, we present the results of a comprehensive image-based characterization study, where a commercial $18650 \mathrm{Li}$-ion battery was investigated across a range of length scales to determine which features could be observed at each level of spatial resolution. Furthermore, a series of 18650 batteries have been imaged before and after applying hundreds of charge cycles, revealing cracks and defects that nucleated as a function of the lithiation/delithiation processes. Examples of these results will be presented alongside a brief description of the technology, as well as a discussion of recent correlative microscopy results that serve as an extension to the XRM technique to provide complementary information on the batteries.

References:

[1] A P Merkle and J Gelb, Microscopy Today (2013) pp. 10-15.

[2] D S Eastwood et. al., Advanced Energy Materials 4 (2013) p. 1-7. 

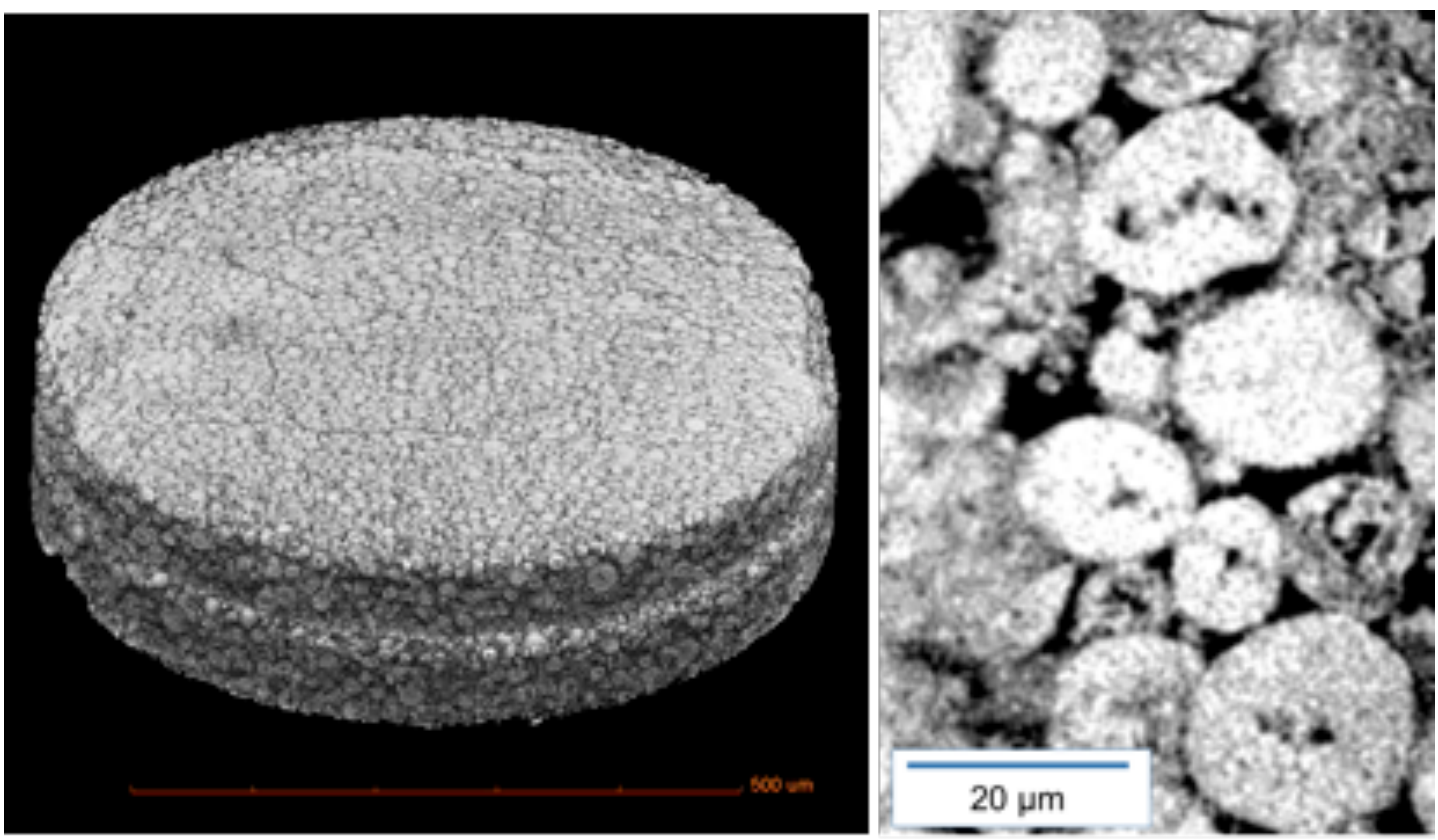

Figure 1. 3D multi-scale imaging with XRM revealed the particles and pore networks, as well as preexisting cracks and voids within the active particles.

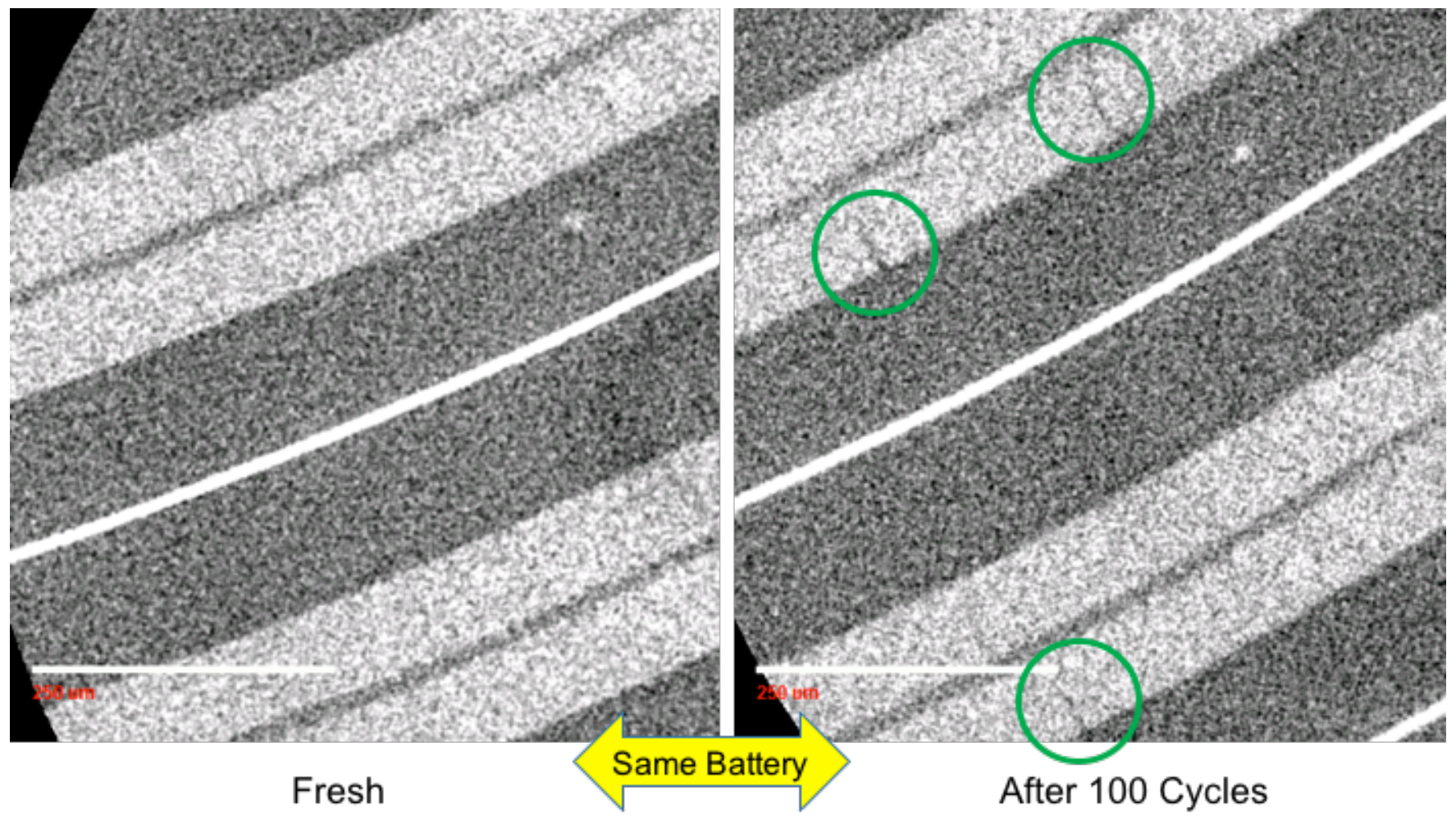

Figure 2. After applying 100 charge cycles and measuring a capacity fade of $\sim 6 \%$, cracks within the positive electrode layer were observed in the outer side of the wrapping (under tensile load during volumetric expansion/contraction of the active particles). 